\title{
Long-Term Time Series Forecasting and Updates on Survival Analysis of Glioblastoma Multiforme: A 1975-2018 Population-Based Study
}

\author{
Georgios Alexopoulos $^{\mathrm{a}, \mathrm{b}}$ Justin Zhang ${ }^{\mathrm{b}}$ loannis Karampelas ${ }^{\mathrm{c}}$ Mayur Patel ${ }^{\mathrm{b}}$ \\ Joanna Kemp ${ }^{a, b}$ Jeroen Coppens ${ }^{a, b}$ Tobias A. Mattei ${ }^{a, b} \quad$ Philippe Mercier $^{a, b}$ \\ ${ }^{a}$ Department of Neurosurgery, Saint Louis University Hospital, St. Louis, MO, USA; bSchool of Medicine, Saint Louis \\ University, St. Louis, MO, USA; 'Department of Neurosurgery, Banner Neurological Surgery Clinic, Greeley, CO, USA
}

\section{Key Messages}

- The annual glioblastoma multiforme (GBM) incidence rates will continue to increase by almost $50 \%$ in the upcoming 30 years.

- Accelerated Failure Time (AFT) lognormal distribution best describes the GBM-specific survival pattern, and as an inherent population characteristic, it should be implemented by researchers for future studies. Among various demographic factors, all patients older than 30 years have a poor prognosis, with the age-group $>70$ years old having the worst overall survival. Annual income $>$ USD 75,000 and supratentorial tumor location are favorable prognostics, while surgical intervention provides the highest survival benefit among patients with GBM.

- Cox regression analysis should not be utilized for time-to-event predictions in GBM survival statistics. When compared to the best fit AFT lognormal model, multivariate Cox regression erroneously associated the following factors with GBM-specific survival: infratentorial tumors, nonmetropolitan areas, and White patient race.

- In contrast with what previous Cox regression studies have reported, the demographics such as gender, race, and county type should not be considered as meaningful prognostics when designing future trials.

\section{Keywords}

Epidemiologic studies · Glioblastoma incidence · Survival analysis · Population-based study - Time series forecasting • Glioblastoma multiforme

\footnotetext{
Abstract

Objective: Glioblastomas multiforme (GBMs) are the most common primary CNS tumors. Epidemiologic studies have investigated the effect of demographics on patient survival, but the literature remains inconclusive. Methods: This study included all adult patients with intracranial GBMs reported
}

in the surveillance epidemiology and end results (SEER)-9 population database (1975-2018). The sample consisted of 32,746 unique entries. We forecast the annual GBM incidence in the US population through the year 2060 using time series analysis with autoregressive moving averages. A survival analysis of the GBM-specific time to death was also performed. Multivariate Cox proportional hazards $(\mathrm{PH})$ regression revealed frank violations of the $\mathrm{PH}$ assumption for multiple covariates. Parametric models best described the GBM population's survival pattern; the results were compared to the semi-parametric analysis and the published literature. Results: We predicted an increasing GBM incidence,
Correspondence to:

Georgios Alexopoulos, alexopoulos_george@ hotmail.com 
which demonstrated that by the year 2060 , over 1,800 cases will be reported annually in the SEER. All eight demographic variables were significant in the univariable analysis. The calendar year 2005 was the cutoff associated with an increased survival probability. A male survival benefit was eliminated in the year-adjusted Cox. Infratentorial tumors, nonmetropolitan areas, and White patient race were the factors erroneously associated with survival in the multivariate Cox analysis. Accelerated Failure Time (AFT) lognormal regression was the best model to describe the survival pattern in our patient population, identifying age $>30$ years old as a poor prognostic and patients $>70$ years old as having the worst survival. Annual income >USD 75,000 and supratentorial tumors had good prognostics, while surgical intervention provided the strongest survival benefit. Conclusions: Annual GBM incidence rates will continue to increase by almost 50\% in the upcoming 30 years. Cox regression analysis should not be utilized for time-to-event predictions in GBM survival statistics. AFT lognormal distribution best describes the GBMspecific survival pattern, and as an inherent population characteristic, it should be implemented by researchers for future studies. Surgical intervention provides the strongest survival benefit, while patient age $>70$ years old is the worst prognostic. Based on our study, the demographics such as gender, race, and county type should not be considered as meaningful prognostics when designing future trials.

(c) 2022 S. Karger AG, Basel

\section{Introduction}

Glioblastoma multiforme (GBM) is the most aggressive diffuse glioma of astrocytic lineage and corresponds to WHO grade IV classification [1-3]. GBMs are the most common primary malignant brain tumors, with an annual incidence of 5.26 per 100,000 individuals or 17,000 new diagnoses per year [1-4]. First-line treatment is complex and consists of maximal safe surgical resection followed by radiotherapy with concurrent temozolomide (TMZ) chemotherapy and 6 cycles of maintenance TMZ [1-6]. Despite the aggressive therapy protocol, patient survival remains poor, as GBM is considered an incurable disease $[2,4,7]$. The median GBM patient survival is currently 12-14 months, and unfortunately, less than $10 \%$ of these patients survive 2 years from diagnosis $[4,5]$.

Many epidemiologic reports have investigated the effect of baseline patient characteristics on GBM prognosis and identified demographic factors associated with a survival benefit. Despite this, the literature remains full of inconclusive results $[2,8-13]$. Hence, further studies are warranted to untangle the complexities of demographic data and baseline patient characteristics on GBM-specific survival. In our report, we utilize historical data from one of the largest epidemiologic databases (surveillance epidemiology and end results [SEER]) [14] to predict the direction of future GBM incidence trends for the upcoming 32 years. Through a comprehensive survival analysis workflow, we further demystify the conflicting results previously reported in the literature. After identifying common misuses of statistical methodology in survival analysis studies, we report the relevant and updated prognostic factors associated with a survival benefit in the GBM population.

\section{Methods}

\section{Data and Study Population}

Data were extracted from the surveillance epidemiology and end results (SEER) database (1975-2018) [14]. The SEER compiles cancer incidence and survival data of 18 registries and covers approximately 34.6 percent of the USA population from academic and nonacademic hospitals. The incidence SEER-9 registry (November 2020 sub 1975-2018) was filtered by "Histology recodebrain groupings" = "Glioblastoma" AND "Age recode" "> 15 years old” AND "Primary-Site” = (C71.0: C71.9). Spinal cord GBMs were excluded from the study. At entry, 32,758 subjects were identified. After filtering the dataset for duplicate "Patient IDs," 12 patient profiles were found in the session, and after these duplicates were removed our final sample consisted of 32,746 unique patient entries. Eight categorical and two continuous variables, "Survival" in months, and "Year of diagnosis," were extracted for each patient record (online suppl. material; for all online suppl. material, see www.karger.com/doi/10.1159/000522611). The transparent reporting of a multivariable prediction model for Individual Prognosis or Diagnosis statement was used for this study.

\section{Long-Term Time Series Forecasting with ARIMA}

We attempted to forecast the future annual GBM incidence in the SEER database 32 years ahead of time (2018-2060), by using time series analysis with autoregressive integrated moving average (ARIMA) models $[15,16]$. Autocorrelation analysis used to estimate for serial dependence and calculate the $p / d / q$ estimates for the ARIMA models, frequency domain analysis to examine for cyclic behavior, and decomposition for seasonal adjustments. The $p, d$, and $q$ variables are nonnegative integers that refer to the order of the autoregressive, integrated, and moving average parts of the model, respectively $[15,16]$. The augmented Dickey-Fuller test was used to test for ARIMA stationarity assumptions. When the time series was found to be nonstationary, we first attempted to difference the set by removing the trend and seasonality to make the series stationary, so the ARIMA assumptions are satisfied. The stationary de-seasonalized data were then modelled first with ARMA $(p / d)$ to compute the optimal lag. The best parameters $p$ and $d$ were calculated using the differenced series autocorrelation plots (ACF, PACF) achieving minimization of the maximum likelihood estimation and Akaike's information criterion (AIC) [16].
76

Neuroepidemiology 2022;56:75-89 DOI: $10.1159 / 000522611$
Alexopoulos/Zhang/Karampelas/Patel/ Kemp/Coppens/Mattei/Mercier 
The final ARIMA $(p / d / q)$ model was converted from the ARMA by testing for significant values using random model parameters and adjusting for autocorrelation lag when present. The residuals of the best ARIMA model were then computed to check for goodness of fit using the Box-Ljung test of independence, and the model's performance was internally validated using a hold-out set [15]. Finally, the seasonal ARIMA model was compared with the deseasonalized ARIMA and the best forecasting model based on the moving averages graph was further deployed for predictions [15, 16].

\section{Survival Analysis}

The cohort included all patients with intracranial GBMs reported between the years of 1975 and 2018 in the SEER database, and the outcome variable was the GBM-specific time to death. The single target event for this study was the cause-specific death classification (death attributable to GBM vs. censored) from the time of diagnosis. The time origin was set as the point at which a subject was originally diagnosed with a GBM, and the time scale was the patient survival in months as reported in the SEER. Patients who died from other causes (non-GBM) and subjects who were alive or lost to follow up at the end of the time scale period were considered as censored for the target event. We had no reason to suspect informative censoring in a large population-based database such as the SEER; informative censoring is analogous to nonignorable missing data, which would bias the analysis. The events were also independent of each other, as duplicate patient ID entries were removed. An important assumption for censoring is that the survival probabilities should be the same for patients who were recruited early and those who were recruited late in the study (cohort effect) $[17,18]$. Given the slightly improved GBM patient survival over the recent years due to advanced treatment modalities [1-5], the "no-cohort effect" assumption for calculating the Kaplan-Meier estimates was fulfilled after stratifying the SEER population into homogeneous groups by enrollment year. Nonparametric approaches were first used to generate unbiased descriptive statistics, in conjunction with semi-parametric or parametric tests when appropriate [17-20]. After identifying the optimal cutoff, we report the year-adjusted survival curves in the different subpopulations (strata). The adjusted survival functions were calculated for each covariate in the subpopulations, and the Kaplan-Meier estimates were plotted for the categorical factors of interest. Rank-based tests, such as the log-rank test, were used to statistically test the difference between the Kaplan-Meier survival curves [18]. The semiparametric Cox Proportional model was then used for univariable and multivariate regression analysis in the entire SEER population to estimate the effect sizes by calculating the hazard ratios (HRs) $[17,18]$. The stratified Cox regression model was adjusted for the year of enrollment by adding interaction terms. The proportional hazards $(\mathrm{PH})$ assumption necessitates a constant relationship between the outcome and the covariate vectors over time, and therefore, it is vital for interpretation the Cox regression model [18-21]. The $\mathrm{PH}$ assumption was frankly violated for multiple covariates in the GBM population, and proportionality was unable to be achieved after multiple stratification attempts. As such, we then analyzed the survival probability of GBM patients in the SEER population using fully parametric approaches. In parametric analysis, the outcome is assumed to follow a known distribution, as such, the effect sizes can be expressed either as PHs or accelerated failure time models [22]. Parametric survival analysis approaches

Forecasting and Updates on Survival Analysis of Glioblastoma Multiforme in our study included the exponential, Weibull, Gompertz, gamma, lognormal, and log-logistic distributions to identify the best GBM survival population pattern $[18,22]$. We used AIC and likelihood ratio tests to assess for relative model goodness of fit followed by the $\log (-\log [S(t)])$ plots to check for model validity and evaluate the pattern of survival estimates against time. Here, we report the multivariate regression analysis results of the best parametric model in conjunction with those extracted from Cox regression analysis and compare our results with the literature.

\section{Software}

All analyses were implemented using the R statistical software, version 4.1.0. The functions from "forecast" and "tseries" packages were used to perform time series forecasting with ARIMA. Nonparametric and semi-parametric survival approaches were completed using the "survival" and "survminer" packages. Parametric distribution model fit was performed using the "flexsurv" package, while Kaplan-Meier estimates and the respective effect sizes from parametric bootstrap simulation were generated using the "survParamSim" implementation in R (https://cran.r-project.org/web/ packages/available_packages_by_name.html).

\section{Results}

\section{Time Series Forecasting with ARIMA}

We can infer from the time series plots that the data points follow an upward trend without any outliers (Fig. 1). Based on graph decomposition of additive series (online suppl.), we identified annual seasonality within the dataset. The population sample satisfied all the ARIMA assumptions except for stationarity, since there was a time-dependent structure without constant variance over time. The augmented Dickey-Fuller test also confirmed a nonstationary dataset $(\mathrm{t}$-stat $=-3.117, p$ value $=0.136)$. After differencing the series, we were able to transform it into a stationary dataset which could be modelled using ARIMA, given that the differenced time series graph now revealed stationary properties without a trend. The autocorrelation plots (ACF, PACF) of differenced series showed alternative positive and negative spikes slowly decaying to zero, without any statistically significant values proving a lag of zero. This means that the annual reported GBM cases in the SEER database are not correlated with each other, and that the elements are random. As such, the best $p$ and $q$ model parameters were selected as 1 for the ARMA $(1,1)$ model. With this approach, our best ARIMA model was computed as the default $\operatorname{ARIMA}(1,1,1)$ or $p=$ $1(p$ value $=0.0039) ; q=1(p$ value $<0.001) ; q=1(p$ value $<0.001)$. The residuals of the best ARIMA revealed a normal distribution with no significant autocorrelation, therefore the model can be used for accurate forecasting (online suppl. material; Box-Ljung $\chi^{2}=4.3473$, $p$ value $=$ 


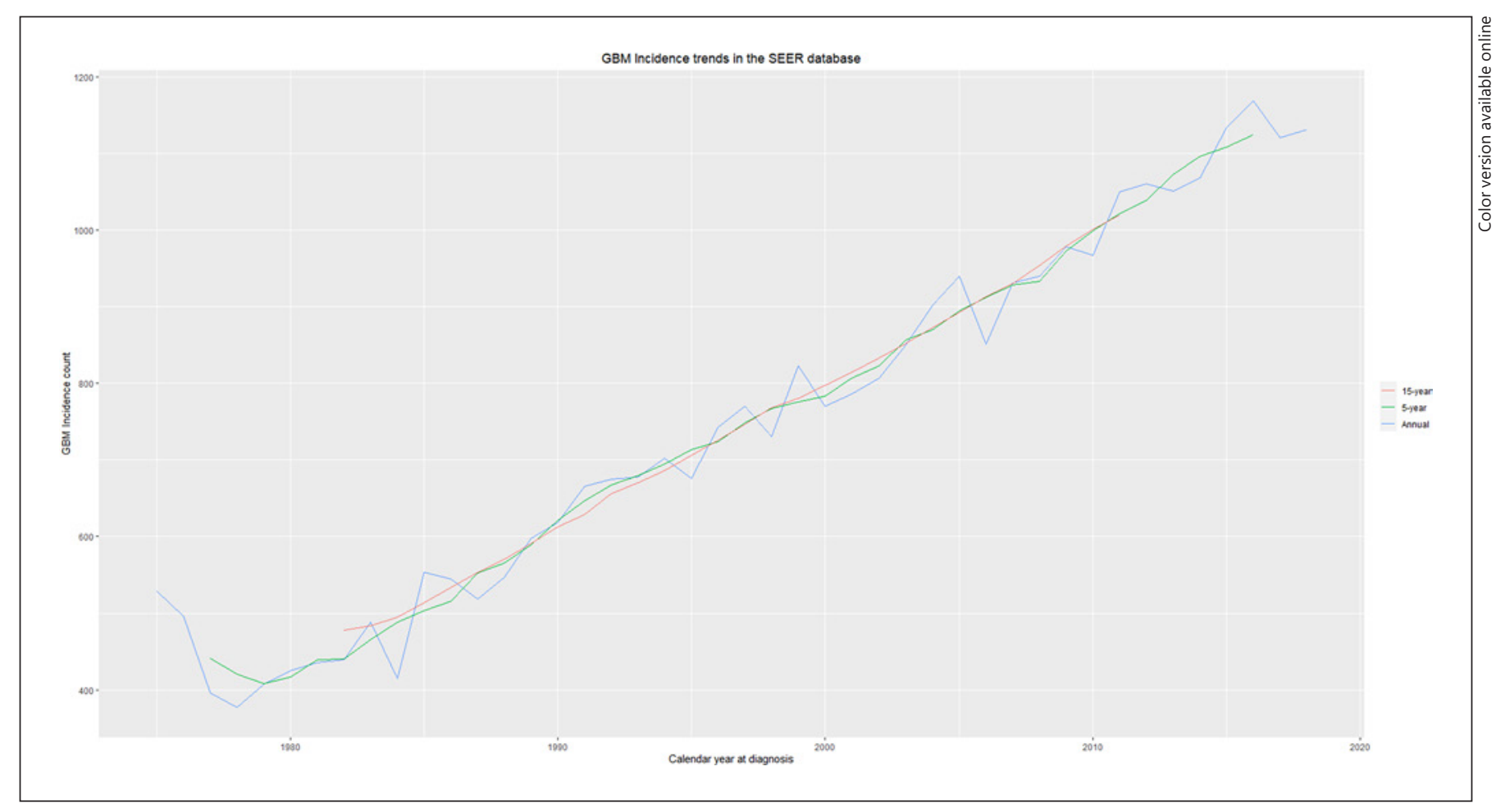

Fig. 1. Moving averages of the GBM incidence rates in the SEER database. Exploratory data analysis. Plots of annual, 5-year, and 15-year moving averages comparing the GBM incidence variances in the SEER. The plots demonstrate the average GBM incidence over the defined time interval.

0.99). When seasonality was added back to the best model, the seasonal ARIMA was even more realistic for predictions based on the moving averages graph.

The parameters of our best seasonal ARIMA $(1,1,1)$ model can be used to accurately forecast the annually reported GBM patient entries in the SEER database. Based on the prediction results (Fig. 2), we demonstrated that by the year of 2060, over 1,800 new GBM cases will be reported annually in the SEER.

\section{Survival Analysis}

Univariable Statistics

Nonparametric Tests. After evaluating the entire SEER population for variations in the GBM patient survival, we identified the calendar year "2005" as the chronologic cutoff, where after 2005, there has been an increased survival in the population (Fig. 3). The median GBM survival time, or the time when the survival probability, $\mathrm{S}(\mathrm{t})$, decreases by $50 \%$, has increased from 6 months prior to 2005 ( $95 \%$ confidence interval (CI) $[6,7] ; p<0.0001)$ to 9 months in our current generation (95\% CI $[9,9]$; $p<0.0001)$. Therefore, the survival curves are reported separately into year- adjusted groups: stratum $\mathrm{A}$ includes all patients who were enrolled in the SEER from 1975 to 2004, and stratum B includes all patients enrolled between the years of 2005 and 2018; the strata contain 18,359 and 14,387 patients, respectively. The side-by-side Kaplan-Meier curves for categorical factors of interest in the two subpopulations are depicted in Figure 4. For the reader, significant comparisons between these two subpopulations cannot be safely made; the curves are only valuable to demonstrate survival trends across time, and stratification was only performed in this study to satisfy the Kaplan-Meier estimator assumptions. The entire population will be tested for covariate significance and effect sizes in the semi-parametric and parametric analyses.

The Kaplan-Meier Estimates of Demographics and Tumor Characteristics on Patient Survival. There is a continuous survival advantage in GBM patients younger than 40 years old, with the highest impact on ages $<30$ years old. The median survival has an increasing trend from 19 months $(95 \% \mathrm{CI}[16,21] ; p<0.0001)$ prior to the year of 2005 to 30 months currently (95\% CI [26, 35]; $p<$ 0.0001 ). Patients older than 70 years of age have the worst 


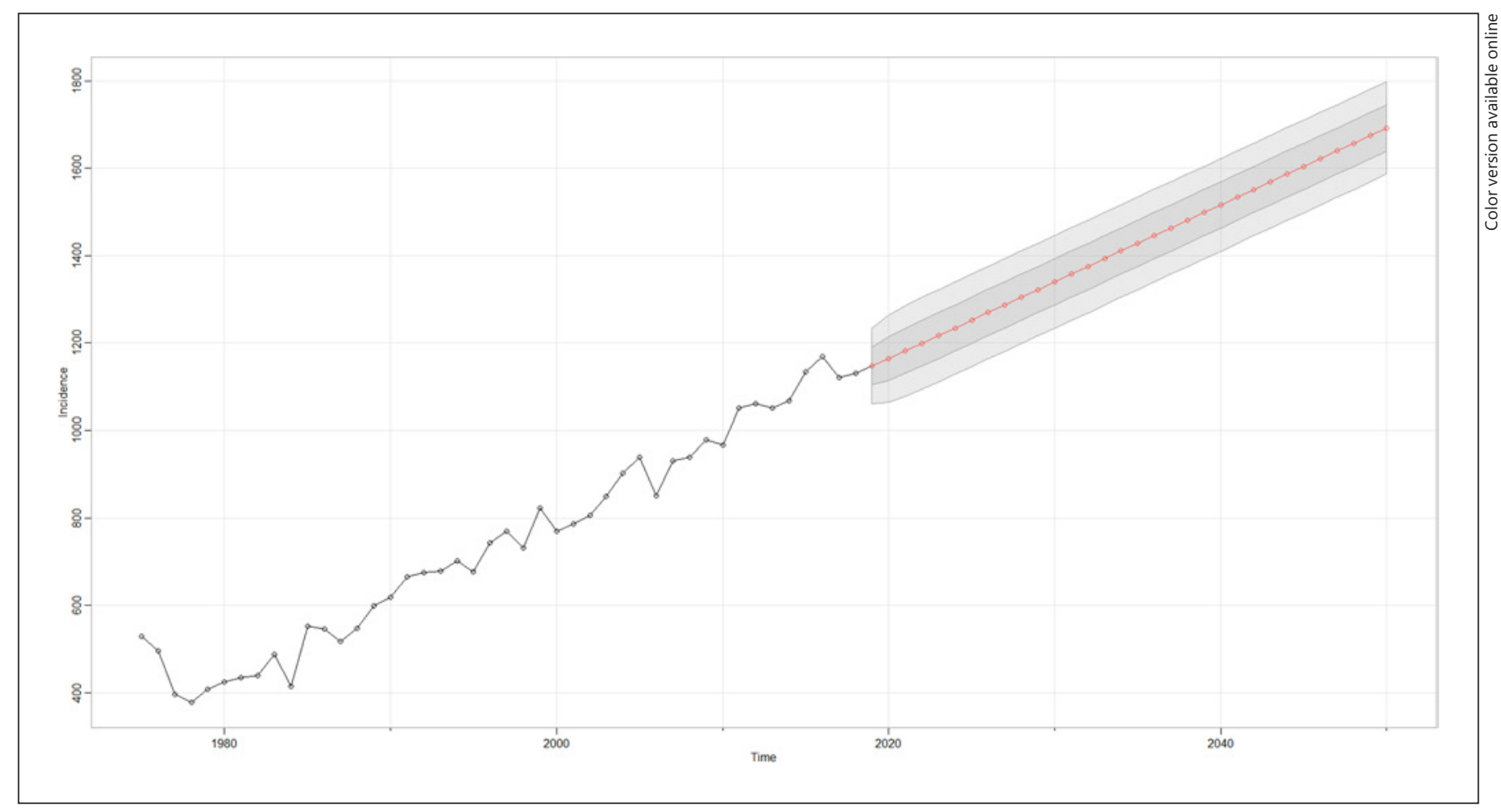

Fig. 2. Forecasts of the annual GBM incidence rates in the SEER database using the best ARIMA $(1,1,1)$ model with added seasonality. As depicted here, the black dots represent the SEER data used to train the model, and the red dots represent the forecast results for the years $2018-2060$, while the $80 \%$ and $95 \%$ prediction inter- vals are shown in grey and light grey colors, respectively. The parameters of the best seasonal ARIMA $(1,1,1)$ model can be used to accurately forecast the annually reported GBM incidence in the database. By the year of 2060 more than 1,800 new patient entries will be reported annually in the SEER. overall prognosis in both subpopulations. When transitioning from 1975 to 2005, we identified a 3-month median overall increase in the survival probability among all racial groups, with White Caucasians having the worst prognosis in both strata. Even though there is a gradual increase in the survival probability among both genders over time, the significant difference in the median survival of males over females prior to 2005 (Stratum A; $p=$ $0.002)$ ceases to exist in Stratum B $(p=0.2)$, as demonstrated in Figure 4. There has been a steady trend of increased survival among all median household income groups in both subpopulations with a lower survival probability in patient's earning $<$ USD 50,000/year ( $p<$ $0.0001)$. Patients in nonmetropolitan areas were the ones with the lowest survival advantage in both strata: Stratum A, $S(t)=6$ months (95\% CI $[6,7] ; p<0.0001)$, and Stratum $\mathrm{B}, \mathrm{S}(\mathrm{t})=8$ months $(95 \% \mathrm{CI}[7,8] ; p<0.0001)$. We observed an increased tendency in the $\mathrm{S}(\mathrm{t})$ among all GBM tumor locations except for the infratentorial lesions (Fig. 4), while surgical intervention also increased the me- dian survival in both strata $(p<0.0001)$. In the current generation, brainstem GBM locations were associated with the highest survival benefit among primary site groups (Fig. 4).

\section{Univariate Cox Regression versus Stratified Cox by \\ Enrollment Year}

The regression beta coefficients along with the HRs and variable significance in relation to the GBM-specific survival for the entire population were calculated. Each factor was assessed through separate univariate Cox regression analysis followed by Cox models, stratified by enrollment year in the SEER. These models were trained on the entire population of 32,746 patient entries. All agegroups $>40$ years, White race, and nonmetropolitan areas were associated with poorer survival in both models. Surgery, metropolitan areas ( $>1$ million), and median household income $>$ USD 75,000 were associated with improved patient survival. In the year-stratified Cox model, infratentorial GBMs showed the best survival benefit among 


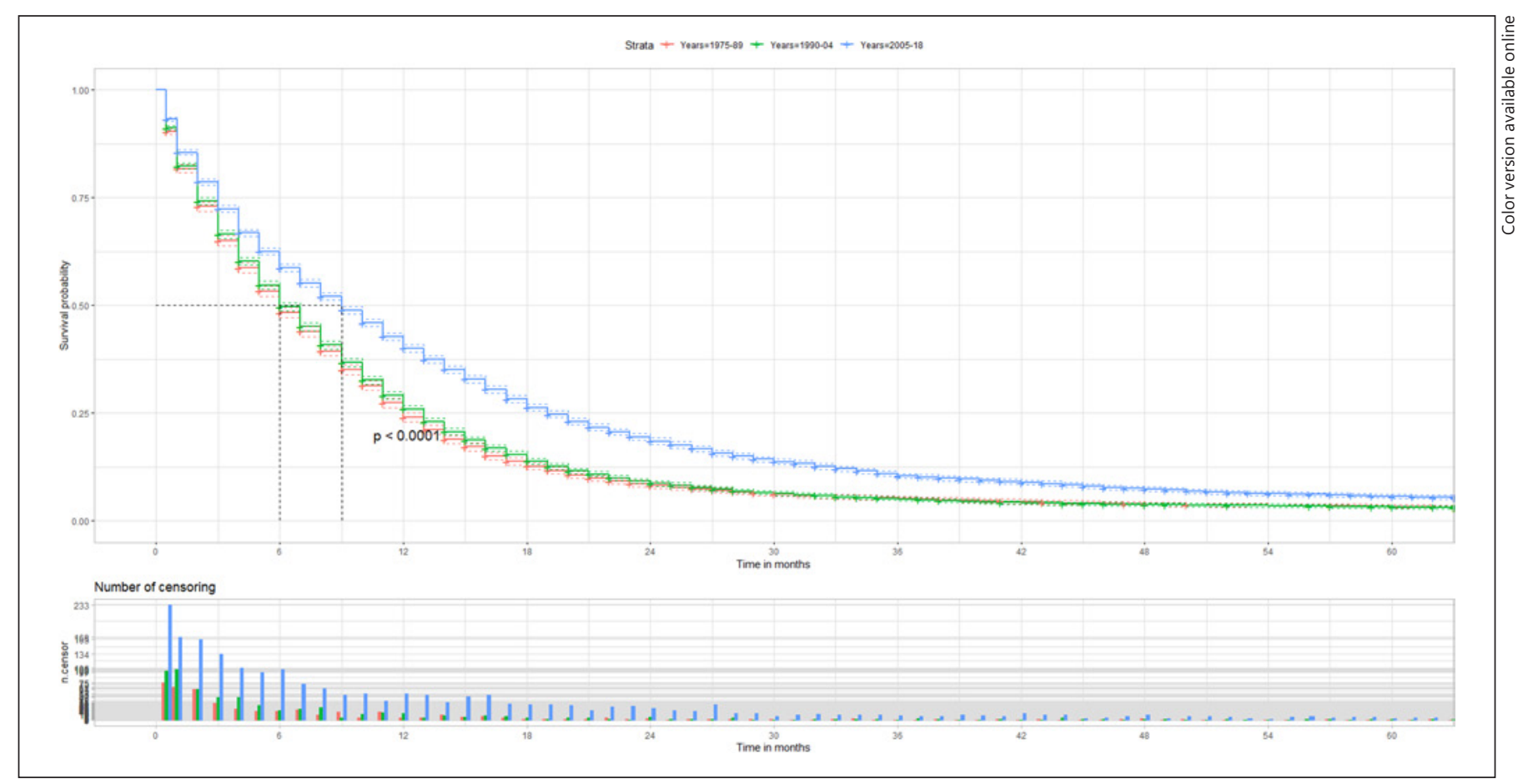

Fig. 3. Kaplan-Meier estimates of GBM patient survival stratified by calendar year at diagnosis. The horizontal axis ( $x$-axis) represents time in months following GBM diagnosis, and the vertical axis ( $y$-axis) shows the survival probability. The colored lines represent survival curves of three distinct subpopulations (19751989, 1990-2004, 2005-2018) along with the respective 95\% CIs in colored dashed lines. The calendar year 2005 is the chronologic cutoff associated with an increased survival in the population. The $p$ value of the Log-Rank comparing the three groups is also demonstrated $(p<0.0001)$. The median survival has increased from 6 months prior to 2005 (95\% CI $[6,7] ; p<0.0001)$ to 9 months in our current generation (95\% CI $[9,9] ; p<0.0001)$. The survival probability for each group is depicted in black dashed lines. The number of censored subjects at time following GBM diagnosis in months is shown in the lower part of the survival plot. primary site groups, reducing the hazard factor by $46 \%$ $(\mathrm{HR}=0.54,95 \%$ CI $[0.39,0.75] ; p<0.0001)$, followed by brainstem GBMs with a HR decrease by $36 \%(\mathrm{HR}=0.64$, $95 \%$ CI $[0.45,0.92] ; p<0.0001)$. The variable male sex was associated with a favorable prognosis only in the univariate $\operatorname{Cox}(\mathrm{HR}=0.96,95 \% \mathrm{CI}[0.94,0.98] ; p<0.0001)$, while this effect was eliminated in the year-adjusted Cox model.

\section{Multivariate Regression Analysis}

The entire population of 32,746 patients was used to build all multivariate models.

Semi-Parametric Models. Cox PHs regression. We fit a Cox regression model using all the significant covariates in the univariable analysis while utilizing interaction terms to stratify by enrollment year and control for treatment effect bias. The HRs for each respective covariate can be seen in Figure 5. The HRs here are interpretable as multiplicative effects on the hazard. When holding the other factors constant, surgical intervention reduces the hazard by a factor of 0.53 or $47 \%$ ( $\mathrm{HR}=0.53,95 \% \mathrm{CI}$ $[0.51,0.54] ; p<0.001)$ and it is the most influential covariate in the model. The effect of sex on patient survival is again eliminated in the multivariate regression, but now all age-groups above 30 years old are strongly associated with worse prognosis. White race shows a weak relationship with an increased risk of death $(\mathrm{HR}=1.11,95 \% \mathrm{CI}$ $[1.05,1.17] ; p<0.001)$, and the GBM locations "brainstem" and "infratentorial" are associated with the highest survival benefit among primary site groups. Patients with a median income higher than USD 75,000 had an approximately $5 \%$ increase in the survival benefit $(\mathrm{HR}=0.95$, $95 \%$ CI $[0.92,0.98] ; p<0.001)$, while residents of nonmetropolitan areas had a $6 \%$ increased hazard. The estimated survival probability at any given point in time is demonstrated in Figure 5.

Proportional hazards assumption. The Schoenfeld residuals test was significant for multiple covariates in the model. The nonproportionality was further supported by 


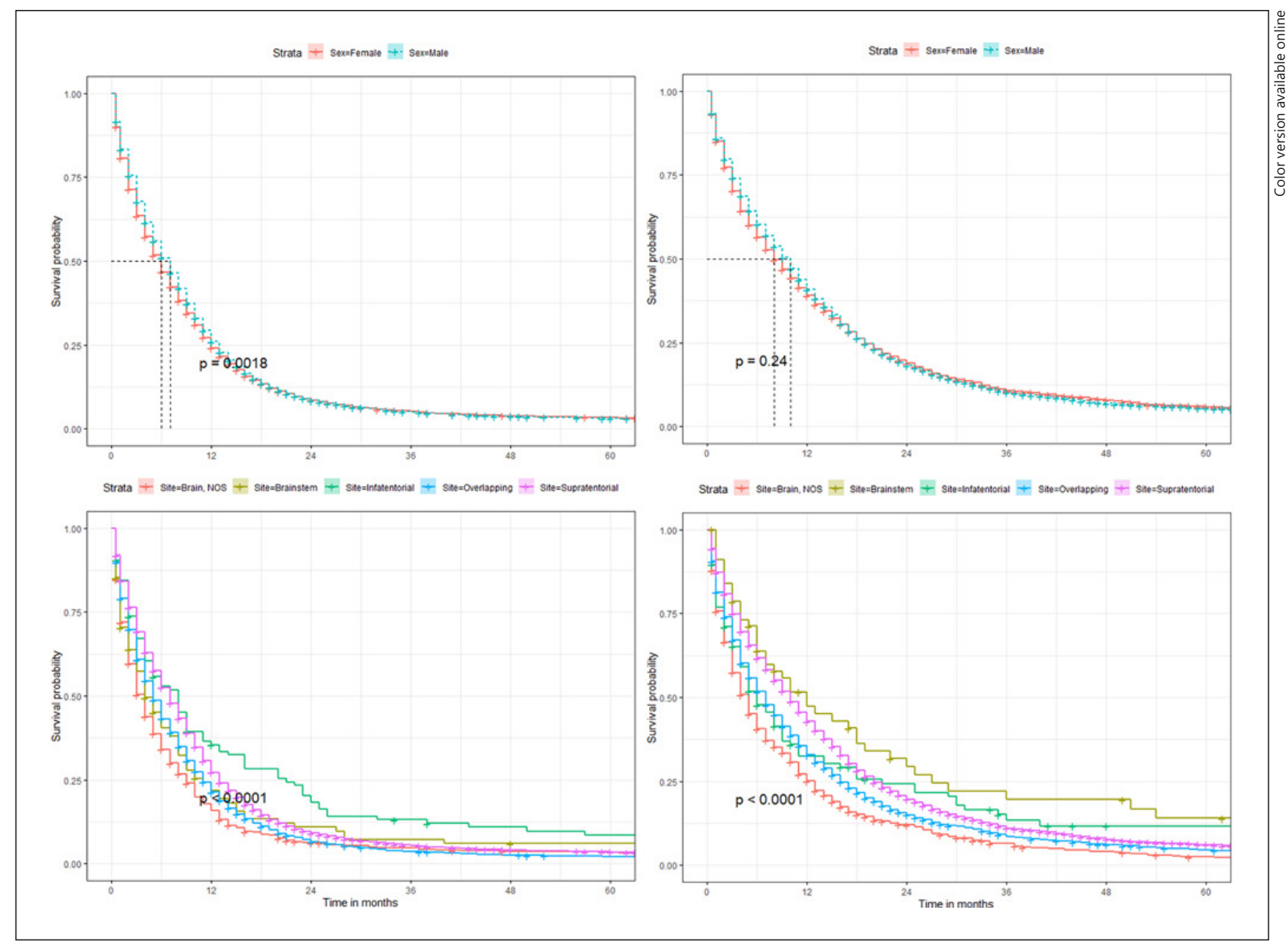

Fig. 4. Side-by-side Kaplan-Meier survival estimates comparing selected variable significance between the two year-adjusted GBM subpopulations (strata). In the left side, stratum A includes all patients enrolled from 1975 to 2004 compared to stratum B (right) including all patients enrolled between the years of 2005 and 2018 in the SEER. The $p$ values of the Log-Rank tests are demonstrated. When transitioning from 1975 to 2005, there is a gradual increase in the survival probability among both genders, but the survival

graphical diagnostics for the above categorical covariables of interests given the $\log (-\log [S(t)])$ plots did not demonstrate any parallelism. Additionally, we were unable to correct for nonproportionality in the models after multiple stratification attempts, and therefore, we assume a frank violation of the $\mathrm{PH}$ assumption for the GBM population. Consequently, Cox regression should not be considered valid for describing the survival characteristics of the GBM population when demographic covariates are utilized.

Forecasting and Updates on Survival Analysis of Glioblastoma Multiforme advantage of males over females prior to 2005 (top left; $p=0.0018$ ) is not evident in chronologic stratum $\mathrm{B}$ (top right; $p=0.24$ ). In the bottom row, infratentorial GBMs provide the highest survival advantage among tumor locations for patients enrolled prior to 2005 (bottom left), and this remains especially evident during the second year of patient survival post GBM diagnosis, while brainstem tumor locations are associated with the highest survival benefit in our generation (bottom right).

Parametric Survival Analysis. Parametric model fit. The lognormal distribution achieved the lowest AIC and likelihood ratio tests relative to other distribution results, therefore, indicating a more parsimonious model able to better describe the GBM population survival pattern. However, AIC allows one to assess the relative fit, so the absolute parametric model goodness of fit for validity was assessed through Q-Q graphical plots, which demonstrated linearity in a function of time for the lognormal model. The lognormal-based cumulative hazard curves also 


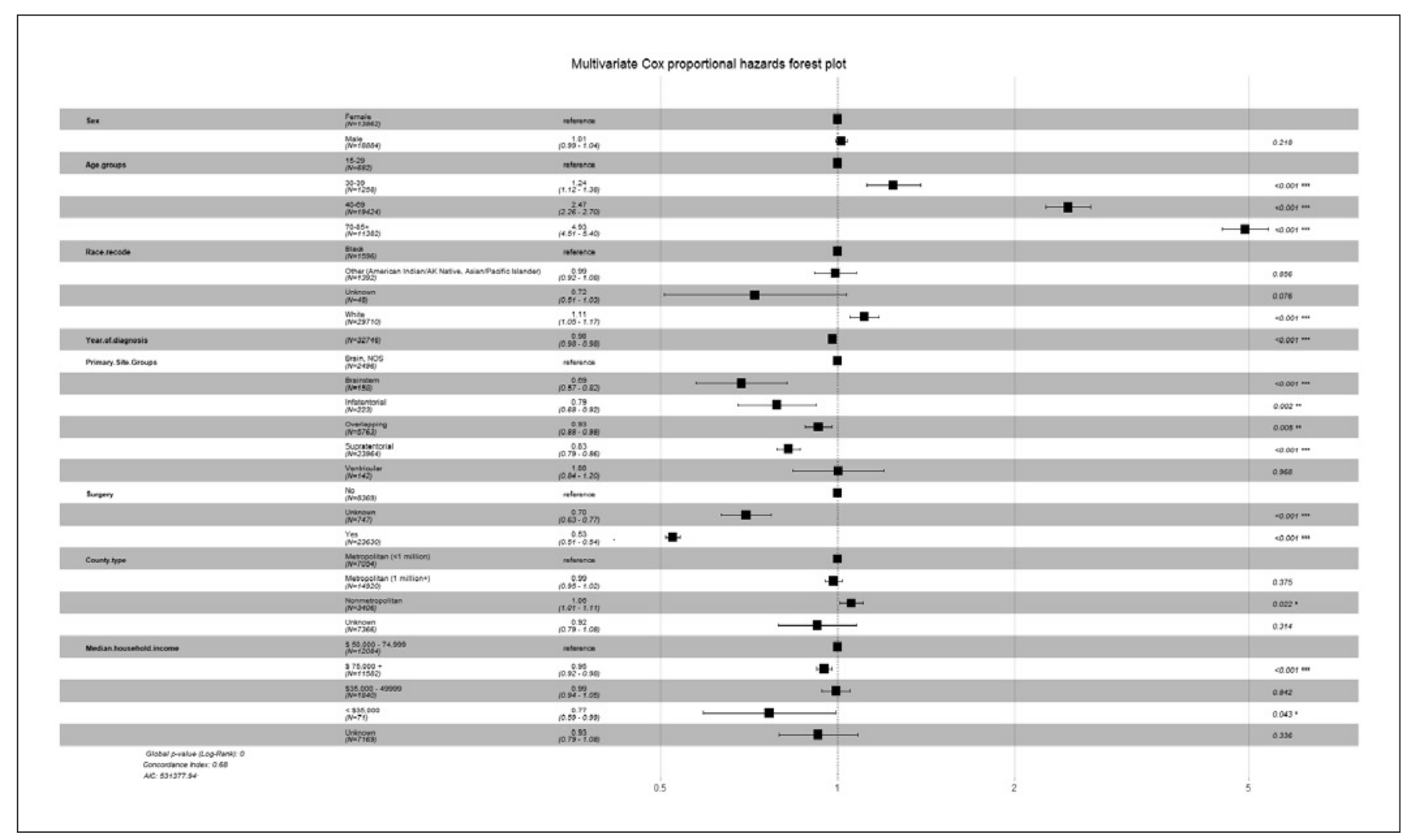

Fig. 5. Forest plot for multivariate Cox PHs model. The HRs for each respective covariate are shown. All significant factors in the univariable regression were included in the multivariate model. The HRs are interpretable as multiplicative effects on the hazard. When holding the other factors constant, surgical intervention is strongly associated with a survival benefit ( $\mathrm{HR}=0.53,95 \% \mathrm{CI}$ $[0.51,0.54])$. All age-groups $>30$ years old are bad prognostics, while GBM patients older than 70 years of age have the highest hazard $(\mathrm{HR}=4.93,95 \% \mathrm{CI}[5.41,5.40]$. There is no effect of sex on

followed through the Kaplan-Meier estimates with a slope of 1 . We conclude that the lognormal distribution best describes the survival pattern in the GBM population.

Accelerated Failure Time Model. The Kaplan-Meier estimate demonstrated a uniform censoring between days 180-500 following the GBM diagnosis, a result that is attributable to a steady study enrollment. After we fit a lognormal model using all the significant variables in the univariable analysis, we identified all patient age-groups $>30$ years old having a negative association with survival, while the factors: supratentorial GBM location, surgical intervention, and median household income >USD 75,000 were good prognostics. The variables gender, race, and county type failed to achieve significance in the para- patient survival, while White race has a weak relationship with an increased risk of death. The GBM locations "brainstem" and "infratentorial" are associated with a survival benefit, even though the respective CIs are wide. A median income $>$ USD 75,000 is a good prognostic, while residents of nonmetropolitan areas have an increased risk of death. There is no effect of sex on GBM-specific survival in the multivariate model. Unfortunately, frank violations of the $\mathrm{PH}$ assumption make Cox regression not valid to analyze the survival pattern in the GBM population. metric multivariate regression (Table 1). In the same model, surgical intervention (95\% CI $[6,7] ; p<0.0001$ ) increased the median survival from 2.9 months (95\% CI $[2.76,3] ; p<0.0001)$ to over 8 months $(95 \%$ CI $[8,8.42]$; $p<0.0001)$. The survival probability decreased from a median of 17.6 months among ages $30-39$ years old ( $95 \%$ CI $[15.8,19.3] ; p<0.0001)$ to 3.4 months $(95 \%$ CI [3.25, 3.45]; $p<0.0001)$ in patients older than 70 years of age (Fig. 6). GBM patients with household income $>$ USD 75,000 had a significant increase in the $S(t)$ with a median of 6.4 months (95\% CI $[6.21,6.64] ; p<0.0001)$. The relationships between the two most influential covariates in the model are demonstrated in Figure 6. 
Table 1. Parametric survival analysis

\begin{tabular}{|c|c|c|}
\hline Predictor variable & $\beta$ coefficient $(95 \% \mathrm{CI})$ & $p$ value \\
\hline \multicolumn{3}{|l|}{ Sex } \\
\hline Male & $0.005(-0.009,0.019)$ & 0.71 \\
\hline Female & $*$ & \\
\hline \multicolumn{3}{|l|}{ Age at diagnosis (years) } \\
\hline $30-39$ & $-0.256(-0.316,-0.196)$ & $<0.0001$ \\
\hline $40-69$ & $-1.05(-1.099,-0.56)$ & $<0.0001$ \\
\hline $70-85+$ & $-1.78(-1.829,-1.731)$ & $<0.0001$ \\
\hline$<30$ & $*$ & \\
\hline \multicolumn{3}{|l|}{ Race } \\
\hline American Indian/Pacific Islander & $0.058(0.011,0.105)$ & 0.215 \\
\hline Unknown & $0.286(0.093,0.479)$ & 0.139 \\
\hline White & $-0.037(-0.07,-0.005)$ & 0.249 \\
\hline African American & $*$ & \\
\hline Calendar year at diagnosis & $0.017(0.016,0.017)$ & $<0.0001$ \\
\hline \multicolumn{3}{|l|}{ Primary site } \\
\hline Brainstem & $0.207(0.104,0.31)$ & 0.044 \\
\hline Infratentorial & $0.049(-0.381,0.136)$ & 0.574 \\
\hline Overlapping & $0.131(0.101,0.161)$ & $<0.0001$ \\
\hline Supratentorial & $0.275(0.248,0.302)$ & $<0.0001$ \\
\hline Ventricular & $-0.135(-0.24,-0.03)$ & 0.201 \\
\hline Brain, not specified & $*$ & \\
\hline \multicolumn{3}{|l|}{ Surgery } \\
\hline Unknown & $0.413(0.355,0.471)$ & $<0.0001$ \\
\hline Yes & $0.811(0.795,0.827)$ & $<0.0001$ \\
\hline No & $*$ & \\
\hline \multicolumn{3}{|l|}{ County } \\
\hline Metropolitan (>1 million) & $0.007(-0.013,0.026)$ & 0.729 \\
\hline Nonmetropolitan & $-0.021(-0.049,0.006)$ & 0.427 \\
\hline Unknown & $-0.049(-0.139,0.042)$ & 0.595 \\
\hline Metropolitan (<1 million) & $*$ & \\
\hline \multicolumn{3}{|l|}{ Median household income } \\
\hline$>$ USD 75,000 & $0.087(0.069,0.104)$ & $<0.0001$ \\
\hline USD 35,000-49,999 & $-0.008(-0.041,0.024)$ & 0.793 \\
\hline$<$ USD 35,000 & $0.169(0.016,0.322)$ & 0.267 \\
\hline Unknown & $0.164(0.073,0.256)$ & 0.073 \\
\hline USD 50,000-74,999 & $*$ & \\
\hline
\end{tabular}

Accelerated failure time model using the lognormal distribution to best describe the survival pattern in the GBM population, SEER-9 from 1975 to $2018(N=32,746)$. Statistically significant associations are demonstrated in bold. * Reference group.

\section{Discussion}

GBM is the most common and lethal tumor of the central nervous system [1-8]. Risk factors include history of radiotherapy, decreased susceptibility to allergy, various immune factors, and specific single nucleotide polymorphisms [1-5]. The natural history of treated GBM remains very poor with 5-year survival rates of 5\% [2-7]. Even though GBMs are the highest funded intracranial malignancies by the American National Institutes of Health, there has been no notable survival improvement in population statistics over the last three decades [2-7].
The 3-year GBM-specific survival has only minimally improved from $8.0 \%$ in 2004 to $10.5 \%(p<0.001)$ currently [9].

\section{Time Series Forecasting for Annual GBM Incidence}

\section{Prediction}

Based on the 2013 Central Brain Tumor Registry of the USA report, the average annual age adjusted GBM incidence rate is $3 \cdot 19 / 100,000$ population $[2,23]$. This is in concordance with the annual reported cases in the SEER [14], with an adjusted frequency of 1,131 new patient entries for the year 2018. Therefore, our best sea- 


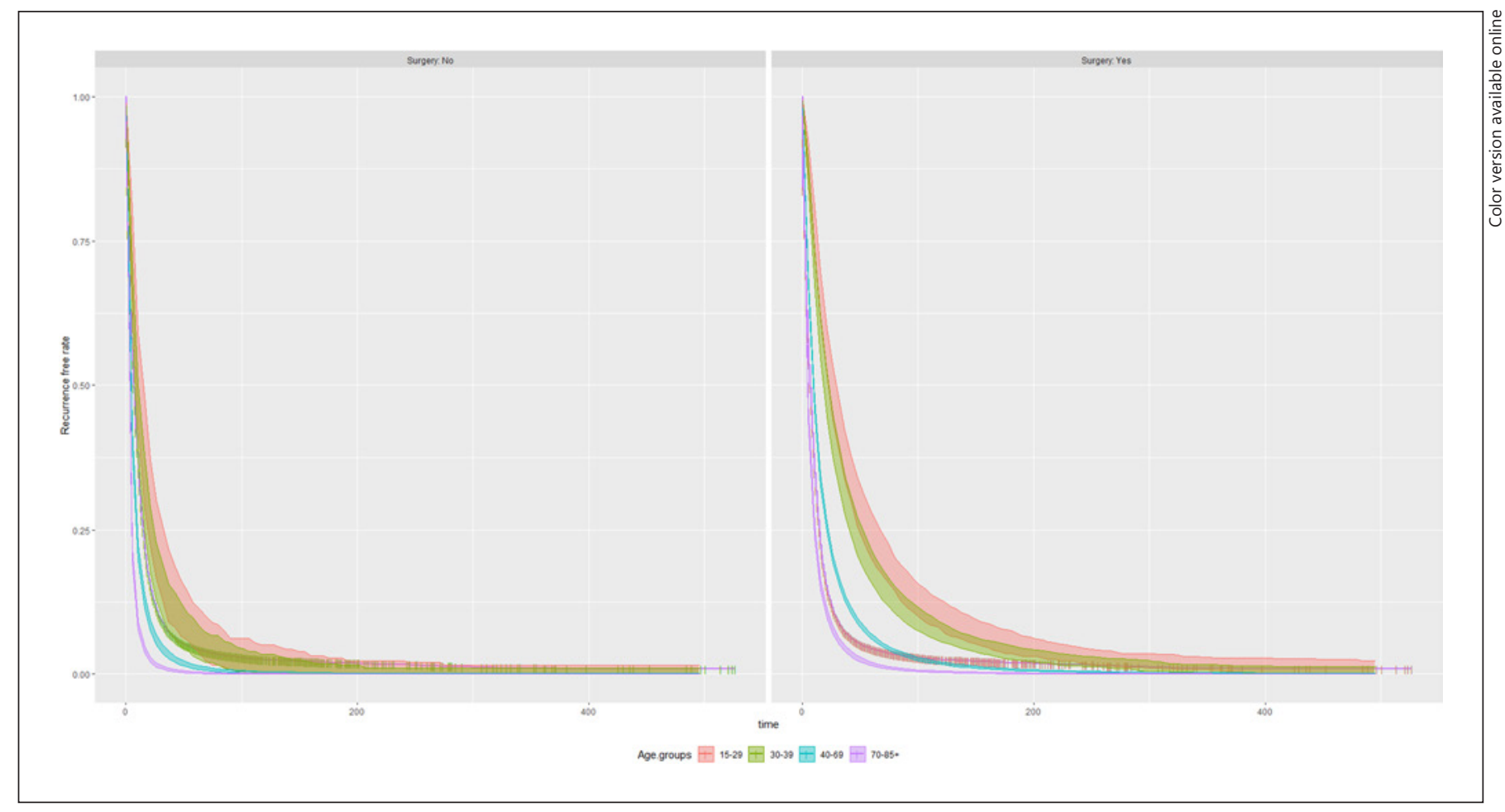

Fig. 6. Generation of Kaplan-Meier curves with prediction intervals from parametric bootstrap simulation of the best fit AFT lognormal model. The relationships between the two most influential covariates in the model are demonstrated. The horizontal axis $(x$ axis) represents time in months following GBM diagnosis, and the vertical axis ( $y$-axis) shows the recurrence free rate. The colored lines represent the survival curves of different patient ages in the population grouped by surgical intervention. All age-groups $>30$

sonal ARIMA model built on the SEER time series data could be used to forecast the annual GBM incidence rates in the US population. Based on the prediction results (Fig. 1), by the year of 2060 , there would be more than 1,800 annual GBM cases reported in the database. Unfortunately, the epidemiologists have previously emphasized this rising GBM incidence trend in the global population $[24,25]$. Our study is the first to indirectly predict the increasing incidence rates by accurately forecasting the annual GBM patient entries in the SEER database 32 years ahead of time (2018-2060). The results could reflect an aging population pattern or a concurrent environmental hazard contributing to the increased annual incidence rates, both of which would be outside of the scope of this study to define. This troubling trend emphasizes more than ever the importance of further research into the GBM etiology, and the need for advances in treatment. The future of the GBM epidemiology will years old are bad prognostic factors, while patients older than 70 years have the worst outcomes $(\beta=-1.78,95 \%$ CI $[-1.829$, $-1.731]$ ). Surgical intervention remains the strongest predictor of survival ( $\beta=0.811,95 \% \mathrm{CI}[0.795,0.827])$ with the highest benefit in the age-groups $<30$ years old. Surgical intervention (95\% CI [6, 7]; $p<0.0001)$ increases the median survival from 2.9 months ( $95 \%$ CI $[2.76,3] ; p<0.0001)$ to over 8 months (95\% CI $[8,8.42] ; p<$ $0.0001)$.

depend on large clinical databases, potentially leading to further understanding of the unknown environmental and genetic hazards contributing to the development of this devastating disease.

\section{Updates on Survival Analysis}

Calendar Year of Diagnosis

The year of 2005 is the chronologic cutoff after which a significantly increased survival was identified in the GBM population. This correlates with previously reported results [2-9], reflecting the introduction of TMZ plus radiotherapy in GBM treatment following the successful Stupp trial in 2005 [26]. Publications in the TMZ era reported a median overall survival of 15.6 months [2-9], but this does not necessarily correlate with the SEER nonparametric survival analysis, which demonstrated a survival probability of 9 months following the initiation of Stupp protocol (95\% CI $[9,9] ; p<0.0001)$. The calendar 
year at diagnosis was a significant covariate in all forms of multivariate analysis in our study.

\section{Sex}

There have been multiple conflicting results about the effects of sex on GBM patient survival [2, 12,23]. Authors previously reported that males have a survival advantage over females in the first-year post diagnosis [2,23]. Conversely, a limited SEER study showed a decreased 5-year GBM-specific survival in males $(6.8 \%)$ when compared to females $(8.3 \%)$. The same study also reported that male patients had the lowest survival rates across different age subgroups $(p=0.002$ univariate and $p<0.001$ in multivariate analysis) [12]. Similarly, Ostrom et al. [23] reported a female survival advantage after analyzing samples collected via both the SEER registry and a multicenter tertiary medical center network. Further expanding these results, other authors emphasized the biologically distinct nature of GBMs among sexes and called for the introduction of sex-targeted approaches to treatment [27]. In our study, we demonstrated how the Kaplan-Meier estimates showed a significant difference in the median survival probability of males over females prior to 2005, which was eliminated after the introduction of the Stupp protocol. A similar phenomenon was seen in our univariable Cox regression analysis ( $\mathrm{HR}=0.96,95 \%$ CI $[0.94,0.98] ; p<$ 0.0001 ), where the effect was eliminated in the year-stratified Cox model. Similarly, there were no significant effects of sex on GBM patient survival in the multivariate models, including the Accelerated Failure Time (AFT) model, which best describes the survival patterns in the GBM population.

\section{Age}

Most epidemiologic studies have identified age at diagnosis as an independent predictor of poor outcomes $[2-7,28,29]$. Increasing patient age has been associated with shortened survival probability [2-7]. Patient age of 50 years at diagnosis has been identified as the appropriate cutoff for the clinical subdivision of GBM patients into prognostically relevant subsets [2-5]. Other authors reported all GBM groups older than 40 years $[28,29]$ as having a decreased survival probability compared to younger individuals. In most studies, however, patients $>70$ years of age have the poorest overall outcomes [2-7, 25]. In contrast, other authors have reported that patient age at the time of GBM diagnosis is not a significant predictor of poorer survival [30,31].

The Kaplan-Meier estimates in our study revealed a continuous survival advantage in GBM patients younger

Forecasting and Updates on Survival Analysis of Glioblastoma Multiforme than 40 years old, with the highest impact on ages $<30$ years of age. All age-groups $>40$ years were associated with poorer survival in the univariable and year-adjusted Cox models. In the multivariate models, all patient groups above 30 years old had a poor prognosis. Of note, in the AFT lognormal model, which best describes the GBM survival pattern, all age-groups $>30$ years old were negatively associated with survival. In this model, the probability further decreased from a median of 17.6 months among ages 30-39 years old to 3.4 months in patients older than 70 years, while GBM patients $>70$ years had the poorest survival among all age-groups.

Race

There is significant controversy regarding the effect of patient race on GBM-specific survival. Liu et al. [8] reported that Asian/Pacific and African American patients possess a survival benefit. Ostrom et al. [23] concluded that Asian Pacific Islanders have increased survival rates compared to both White Caucasians and African Americans at all time points. Multiple authors reported GBM patients of White descent as the ones with the poorest overall survival among all racial groups $[8,11]$. Other reports identified no differences in survival between White, non-Hispanics and Blacks [32]. In addition, populationbased studies have not demonstrated a race-based disparity in GBM survival $[2,7]$.

We demonstrated how the Kaplan-Meier estimates identified White patients as having the worst GBM-specific prognosis in both chronological strata, an effect which persisted in the univariable and age-stratified Cox regression analyses. Similarly, the multivariate Cox model related White race with poorer survival $(\mathrm{HR}=1.11$, $95 \%$ CI $[1.05,1.17] ; p<0.001)$. After reporting the multiple violations of the $\mathrm{PH}$ assumption when Cox regression was utilized to describe the pattern in the GBM population, we here emphasize how we could have misused the statistics in this study if not further assessing the population with parametric survival analysis. In our best fit AFT model, the covariate "race" failed to achieve significance in the multivariate analysis when holding other factors constant.

\section{GBM Tumor Location}

The prognosis of cerebellar GBMs with respect to their supratentorial counterparts remains unclear $[1,2,33]$. However, studies previously reported age-associated differences in the survival probability of younger patients with cerebellar GBMs as compared with supratentorial tumors $[2,33]$. Our survival analysis curves and year- 
stratified Cox model showed cerebellar GBMs as having the best survival benefit among all primary site groups, followed by brainstem GBMs. This effect persisted in the multivariate Cox regression analysis. Again, the best fit AFT model resolved the controversy after identifying supratentorial GBM locations as the only good prognostic among primary site groups.

\section{Surgical Treatment}

Surgical intervention is a good prognostic in multiple epidemiologic reports [1-10, 29]. Higher GBM institutional surgical case volumes have been also associated with improved survival [34]. In our population-based study, surgery provided the highest survival benefit in all forms of statistical analyses, and the AFT model highlighted the covariate "surgical intervention" as the most influential factor on patient survival (Table 1).

\section{Socioeconomic Status}

The economic status of a GBM patient's community may influence survival [1-10]. Aneja et al. [35] reported GBM patients with higher median incomes as being more likely to receive gross total tumor resection after receiving surgical treatment. Bower et al. [36] found a $25 \%$ decreased risk of GBM-specific death in high-income communities. A national cancer database study reported GBM patients with the highest median incomes to have an improved 5-year overall survival [28]. Other authors suggest no relationship between socioeconomic status and GBM-specific survival [37]. Our Kaplan-Meier estimates revealed a lower survival probability for GBM patients earning $<$ USD 50,000/year, while the semi-parametric analysis demonstrated a survival benefit in GBM patients with a median household income $>$ USD 75,000. The AFT model revealed that only patients with a household income $>$ USD 75,000 had increased survival. In contrast, GBM patients in nonmetropolitan areas had the lowest survival probability in the Kaplan-Meier estimates. This effect persisted in both the univariable and multivariate Cox regression analysis, where GBM patients in nonmetropolitan regions had $\sim 6 \%$ increased hazard. Again, the best fit AFT model did not demonstrate any relationship between the covariate "county type" and GBM-specific survival.

\section{Why so Many Conflicting Reports in the Literature?}

Real-world data remain highly complex. The integral quality and unpredictable external validity of each respective dataset could explain some of the variability among the conflicting literature reports. Nevertheless, this should not be the only explanation in large population-based studies, as the conclusions from such reports should be generalizable. Nonparametric models, such as the KM estimates, are limited, in that they do not provide effect sizes and cannot be used to assess the effect of multiple factors of interest at the same time. KM estimates are mainly used for explanatory analyses to simply describe the data with respect to a specific factor. In survival statistics, a well-designed multivariate model remains the only unbiased methodology to control for multiple confounders [16-22]. As such, generalization of univariable analysis results should be done with extreme caution by researchers.

Semi-parametric models are commonly used in the medical research methodology for survival statistics since they are considered less risky [16-20]. Cox regression does not consider a specific probability distribution for the survival time; therefore, the $\mathrm{PH}$ function assumption is an essential model component for accurate survival predictions [16, 38-40]. Cox $\mathrm{PH}$ regression is ubiquitous, and interpretation of the results should not be made when the $\mathrm{PH}$ assumption is frankly violated, especially without any further attempts for stratification or addition of timedependent covariates [16-21]. Whenever the $\mathrm{PH}$ assumption is violated, estimates derived from utilizing the Cox model will lead to an improper fitting and incorrect inferences. AFT models are especially important in such situations, given their parametric distribution for the survival times which can make statistical inference accurate and lead to a proper model fitting. In parametric survival analysis, all parts of the model are specified, both the hazard function and the effect of the covariates on the logarithm of the survival time $[16,22,39]$. Then, the pattern of survival estimates against time is compared to known parametric distributions. Finally, the best parametric distribution to describe the time-to-event pattern is identified, while such distribution is also population specific describing an inherent survival characteristic [39-41]. Disadvantages of the AFT regression are the high complexity and specialized knowledge required to properly assess for population model fit [39-41].

In a previous SEER-based report including GBM patients from 2000 to 2008, Tian et al. [12] utilized multivariate Cox regression models to conclude that gender has a significant effect on patient survival. In another SEER-based study, Ostrom et al. [38] reported similar results, concluding that females have a survival advantage in the GBM population. In that study, Ostrom et al. [38] only reported the nonparametric survival analysis of the KM estimates. Similarly, Gately et al. [30] generalized the
86

Neuroepidemiology 2022;56:75-89 DOI: $10.1159 / 000522611$
Alexopoulos/Zhang/Karampelas/Patel/ Kemp/Coppens/Mattei/Mercier 
multivariate Cox regression results by concluding that age standalone is not a predictor for survival in GBM patients. Liu et al. [8] identified in their Cox regression model that Asian and African American patients had a survival benefit in the GBM population. In all these studies, however $[8,12,30]$, the authors did not seem to assess for proportionality in their Cox regression analysis, as it was inferred from the reports. In a national cancer database study, Cantrell et al. [28] compared the multivariable logistic regression analysis results of two different GBM subpopulations to identify long-term patient survivors. However, when comparing the results between two different subpopulations, safe conclusions cannot be accurately made. Furthermore, stratification prior to fitting the data to the regression algorithms greatly decreases the statistical power in the study. The authors in the same study [28] utilized plain logistic regression to describe the survival probability in the GBM population. This assumes that such a distribution can be predicted by a normal distribution pattern, an assumption that may result in misleading estimates [16-20].

\section{The Value of Parametric Regression on GBM-Specific} Survival Data

AFT models are reliable alternatives to Cox regression, and one of the few available substitutes for survival statistics when the $\mathrm{PH}$ assumption is violated [16$21,39-41]$. In a report that strongly supports the findings in our study, Senders et al. [13] concluded that only AFT algorithms are capable of modeling time-to-event data in GBM survival statistics. Here, we showed how a semi-parametric survival approach would result in misinterpretations when utilized to describe GBM-specific survival data, and we introduce an AFT lognormal distribution as the best overall pattern to predict the survival events in the GBM population. Furthermore, such a pattern is an inherent survival characteristic of the population, concluding that similar AFT lognormal models should be utilized by researchers when designing future clinical trials to assess time-to-event predictions in GBM survival statistics. Based on our study, the demographics: gender, race, and county type should not be considered as meaningful prognostics when designing future trials.

\section{Limitations}

The primary limitations of this study are its integral data quality and the retrospective design. Although all essential factors were extracted from the SEER database to mitigate the risk of confounding, the possibility of influ- ence from unmeasured confounders cannot be excluded. Real-world data are highly complex and an incomplete reflection of reality. There is always a chance for introduction of unpredictable outliers even with basic structural data collection. Forecasting may not be generalizable in the presence of such outliers, given that in realworld scenarios where conditions change, trained models tend to perform poorly. Because our results were extracted from thousands of patients from multiple institutions across the U.S., we expect the fitted models to be more generalizable. Randomized controlled trials would be ideal; however, it is neither practical nor feasible to establish a cohort on this scale. In addition, it is ethically unjustifiable to randomize newly diagnosed GBM patients to a nonsurgical placebo arm to assess for covariate importance.

\section{Conclusions}

Annual GBM incidence rates will continue to increase by almost $50 \%$ by the year of 2060 . Generalization of univariable statistics should be made with extreme caution in survival analysis. Cox regression should not be utilized for time-to-event predictions in GBM survival statistics. AFT lognormal distribution best describes the GBM specific survival pattern, and as an inherent population characteristic, it should be implemented by researchers for future studies. When compared to the best fit AFT lognormal model, multivariate Cox regression erroneously associated the following factors with GBM-specific survival: infratentorial tumors, nonmetropolitan areas, and White patient race. Multivariate AFT parametric models identified all patient age-groups $>30$ years old as having a poor prognosis, with those older than 70 years old as having the worst overall survival. Annual income $>$ USD 75,000 along with supratentorial tumors are favorable prognostics, and surgical intervention provides the highest survival benefit among GBM patients. Based on our study, the demographics such as gender, race, and county type should not be considered as meaningful prognostics when designing future trials.

\section{Statement of Ethics}

The study did not require ethics approval as the information is freely available in the SEER public domain. The public SEER datasets are anonymized; therefore, informed consent was not applicable.
Forecasting and Updates on Survival Analysis of Glioblastoma Multiforme
Neuroepidemiology 2022;56:75-89 DOI: $10.1159 / 000522611$ 


\section{Conflict of Interest Statement}

None declared. No potential conflict of interest related to this study is reported by the authors.

\section{Funding Sources}

No funding was received to complete the study.

\section{Author Contributions}

G.A. was the principal investigator who oversaw the study. G.A. developed all study materials including the trial protocol and study idea, data analysis, interpreted the results, and performed the data collection. G.A. wrote and revised the manuscript. J.Z., M.P., assisted with the data collection and helped write the manuscript. I.K., J.K., J.C., T.M., and P.M. revised the final manuscript. The corresponding author (G.A.) had full access to all the data in the study and had final responsibility for the decision to submit for publication. All authors read and approved the final manuscript.

\section{Data Availability Statement}

The datasets generated and analyzed in the current study are available in the SEER (www.seer.cancer.gov) Database: Incidence - SEER Research Data, 9 Registries, November 2020 Sub (19752018), Surveillance Research Program, released April 2021, based on the November 2020 submission.

\section{References}

1 Tamimi AF, Juweid M. Chapter 8 epidemiology and outcome of glioblastoma. In: De Vleeschouwer S, editor. Glioblastoma [Internet]. Brisbane, AU: Codon Publications; 2017 Sep 27. Available from: https://www.ncbi. nlm.nih.gov/books/NBK470003/.

2 Thakkar JP, Dolecek TA, Horbinski C, Ostrom QT, Lightner DD, Barnholtz-Sloan JS, et al. Epidemiologic and molecular prognostic review of glioblastoma. Cancer Epidemiol Biomarkers Prev. 2014 Oct;23(10):1985-96.

3 Delgado-López PD, Corrales-García EM. Survival in glioblastoma: a review on the impact of treatment modalities. Clin Transl Oncol. 2016 Nov; 18(11):1062-71.

4 Omuro A, DeAngelis LM. Glioblastoma and other malignant gliomas: a clinical review. JAMA. 2013 Nov 6;310(17):1842-50.

5 Gately L, McLachlan SA, Dowling A, Philip J. Life beyond a diagnosis of glioblastoma: a systematic review of the literature. J Cancer Surviv. 2017 Aug;11(4):447-52.

6 Marton E, Giordan E, Siddi F, Curzi C, Canova G, Scarpa B, et al. Over ten years overall survival in glioblastoma: a different disease? J Neurol Sci. 2020 Jan 15;408:116518.

7 Marenco-Hillembrand L, Wijesekera O, Suarez-Meade P, Mampre D, Jackson C, Peterson J, et al. Trends in glioblastoma: outcomes over time and type of intervention: a systematic evidence-based analysis. J Neurooncol. 2020 Apr;147(2):297-307.

8 Liu EK, Yu S, Sulman EP, Kurz SC. Racial and socioeconomic disparities differentially affect overall and cause-specific survival in glioblastoma. J Neurooncol. 2020 Aug; 149(1):55-64.

9 Zreik J, Moinuddin FM, Yolcu YU, Alvi MA, Chaichana KL, Quinones-Hinojosa A, et al. Improved 3-year survival rates for glioblastoma multiforme are associated with trends in treatment: analysis of the national cancer database from 2004 to 2013. J Neurooncol. 2020 May;148(1):69-79.
10 Goryaynov SA, Gol'dberg MF, Golanov AV, Zolotova SV, Shishkina LV, Ryzhova MV, et al. Fenomen dlitel'noil vyzhivaemosti patsientov s glioblastomami. Chast' I: rol' klinikodemograficheskikh faktorov i mutatsii IDH1 $(\mathrm{R} 132 \mathrm{H})$ [The phenomenon of long-term survival in glioblastoma patients. Part I: the role of clinical and demographic factors and an IDH1 mutation (R $132 \mathrm{H}$ )]. Zh Vopr Neirokhir Im N N Burdenko. 2017;81(3):5-16.

11 Patel NP, Lyon KA, Huang JH. The effect of race on the prognosis of the glioblastoma patient: a brief review. Neurol Res. 2019 Nov; 41(11):967-71.

12 Tian M, Ma W, Chen Y, Yu Y, Zhu D, Shi J, et al. Impact of gender on the survival of patients with glioblastoma. Biosci Rep. 2018 Nov 7; 38(6):BSR20180752.

13 Senders JT, Staples P, Mehrtash A, Cote DJ, Taphoorn MJB, Reardon DA, et al. An online calculator for the prediction of survival in glioblastoma patients using classical statistics and machine learning. Neurosurgery. 2020 Feb 1;86(2):E184-92.

14 Surveillance, Epidemiology, and End Results (SEER) Program (www.seer.cancer.gov) SEER*Stat Database: Incidence - SEER research data, 9 Registries, Nov 2020 Sub (1975-2018) - linked to county attributes time dependent (1990-2018) income/rurality, 1969-2019 Counties, National Cancer Institute, DCCPS, Surveillance Research Program, released April 2021, based on the November 2020 submission.

15 Stoffer DS, Shumway RH. Time series: a data analysis approach using R. Taylor \& Francis Group; 2019.

16 Hyndman RJ, Athanasopoulos G. Forecasting: principles and practice. 2nd ed. OTexts; 2018.

17 Fisher LD, Lin DY. Time-dependent covariates in the Cox proportional-hazards regression model. Annu Rev Public Health. 1999;20: $145-57$.
18 Clark TG, Bradburn MJ, Love SB, Altman DG. Survival analysis part I: basic concepts and first analyses. Br J Cancer. 2003 Jul 21; 89(2):232-8.

19 Ng'andu NH. An empirical comparison of statistical tests for assessing the proportional hazards assumption of Cox's model. Stat Med. 1997 Mar 30;16(6):611-26.

20 Huang CY, Ning J, Qin J. Semiparametric likelihood inference for left-truncated and right-censored data. Biostatistics. 2015 Oct; 16(4):785-98.

21 Bellera CA, MacGrogan G, Debled M, de Lara CT, Brouste V, Mathoulin-Pélissier S. Variables with time-varying effects and the Cox model: some statistical concepts illustrated with a prognostic factor study in breast cancer. BMC Med Res Methodol. 2010;10:20.

22 Jackson CH. flexsurv: a platform for parametric survival modeling in R. J Stat Softw. 2016 May 12;70:i08.

23 Ostrom QT, Gittleman H, Farah P, Ondracek A, Chen Y, Wolinsky Y, et al. CBTRUS statistical report: primary brain and central nervous system tumors diagnosed in the United States in 2006-2010. Neuro Oncol. 2013 Nov; 15(Suppl 2):ii1-56.

24 Korja M, Raj R, Seppä K, Luostarinen T, Malila N, Seppälä M, et al. Glioblastoma survival is improving despite increasing incidence rates: a nationwide study between 2000 and 2013 in Finland. Neuro Oncol. 2019;21(3): $370-9$.

25 Miranda-Filho A, Piñeros M, Soerjomataram I, Deltour I, Bray F. Cancers of the brain and CNS: global patterns and trends in incidence. Neuro Oncol. 2017;19(2):270-80.

26 Stupp R, Mason WP, Van den Bent MJ, Weller M, Fisher B, Taphoorn MJ, et al. Radiotherapy plus concomitant and adjuvant temozolomide for glioblastoma. N Engl J Med. 2005 Mar 10;352(10):987-96.

Alexopoulos/Zhang/Karampelas/Patel/ Kemp/Coppens/Mattei/Mercier
Neuroepidemiology 2022;56:75-89

DOI: $10.1159 / 000522611$ 
27 Yang W, Warrington NM, Taylor SJ, Whitmire P, Carrasco E, Singleton KW, et al. Sex differences in GBM revealed by analysis of patient imaging, transcriptome, and survival data. Sci Transl Med. 2019;11(473):eaao5253.

28 Cantrell JN, Waddle MR, Rotman M, Peterson JL, Ruiz-Garcia H, Heckman MG, et al. Progress toward long-term survivors of glioblastoma. Mayo Clin Proc. 2019 Jul;94(7): 1278-86.

29 Tykocki T, Eltayeb M. Ten-year survival in glioblastoma. A systematic review. J Clin Neurosci. 2018 Aug;54:7-13.

30 Gately L, Collins A, Murphy M, Dowling A. Age alone is not a predictor for survival in glioblastoma. J Neurooncol. 2016 Sep;129(3): 479-85.

31 Berger K, Turowski B, Felsberg J, Malzkorn B, Reifenberger G, Steiger HJ, et al. Age-stratified clinical performance and survival of patients with IDH-wildtype glioblastoma homogeneously treated by radiotherapy with concomitant and maintenance temozolomide. J Cancer Res Clin Oncol. 2021 Jan; 147(1):253-62.
32 Barnholtz-Sloan JS, Maldonado JL, Williams VL, Curry WT, Rodkey EA, Barker FG 2nd, et al. Racial/ethnic differences in survival among elderly patients with a primary glioblastoma. J Neurooncol. 2007 Nov;85(2):171-80.

33 Jeswani S, Nuño M, Folkerts V, Mukherjee D, Black KL, Patil CG. Comparison of survival between cerebellar and supratentorial glioblastoma patients: surveillance, epidemiology, and end results (SEER) analysis. Neurosurgery. 2013 Aug;73(2):240-6.

34 Raj R, Seppä K, Luostarinen T, Malila N, Seppälä M, Pitkäniemi J, et al. Disparities in glioblastoma survival by case volume: a nationwide observational study. J Neurooncol. 2020 Apr;147(2):361-70.

35 Aneja S, Khullar D, Yu JB. The influence of regional health system characteristics on the surgical management and receipt of post operative radiation therapy for glioblastoma multiforme. J Neurooncol. 2013 May;112(3): 393-401.

36 Bower A, Hsu FC, Weaver KE, Yelton C, Merrill $\mathrm{R}$, Wicks $\mathrm{R}$, et al. Community economic factors influence outcomes for patients with primary malignant glioma. Neurooncol Pract. 2020 Jul;7(4):453-60.
37 Kasl RA, Brinson PR, Chambless LB. Socioeconomic status does not affect prognosis in patients with glioblastoma multiforme. Surg Neurol Int. 2016 May 6;7(Suppl 11):S282-90

38 Ostrom QT, Rubin JB, Lathia JD, Berens ME, Barnholtz-Sloan JS. Females have the survival advantage in glioblastoma. Neuro Oncol. 2018;20(4):576-7.

39 Swindell WR. Accelerated failure time models provide a useful statistical framework for aging research. Exp Gerontol. 2009;44(3):190200.

40 Basu A, Manning WG, Mullahy J. Comparing alternative models: log versus Cox proportional hazard? Health Econ. 2004 Aug;13(8): 749-65.

41 Zare A, Hosseini M, Mahmoodi M, Mohammad K, Zeraati H, Holakouie Naieni K. A comparison between accelerated failure-time and Cox proportional hazard models in analyzing the survival of gastric cancer patients. Iran J Public Health. 2015;44(8):1095-102. 